\title{
Renal cell carcionoma with synchronous metastases to the bladder and lung
} \author{
Chan $\mathrm{Choi}^{2}$ \\ ${ }^{1}$ Department of Urology, Chonnam National University Medical School, Gwangju, South Korea; \\ ${ }^{*}$ Corresponding Author: urohwang@gmail.com \\ ${ }^{2}$ Department of Pathology, Chonnam National University Medical School, Gwangju, South Korea
}

Won Seok Jang ${ }^{1}$, In Sang Hwang ${ }^{1}$, Eu Chang Hwang ${ }^{1^{*}}$, Seung II Jung ${ }^{1}$, Dong Deuk Kwon ${ }^{1}$,

Received 21 February 2013; revised 28 March 2013; accepted 15 April 2013

Copyright (C) 2013 Won Seok Jang et al. This is an open access article distributed under the Creative Commons Attribution License, which permits unrestricted use, distribution, and reproduction in any medium, provided the original work is properly cited.

\begin{abstract}
Renal cell carcinoma can metastasize to virtually any organ, yet synchronous metastasis to the bladder is extremely rare. A 77-year-old woman presented with gross hematuria. Abdominal and chest computed tomography showed a bilateral renal masses, bladder dome mass, and multiple lung metastasis. Transurethral resection of the bladder tumor and sonography guided renal biopsy were performed. Both pathology diagnoses were clear cell renal cell carcinoma. Targeted therapy using pazopanib was administered to the patient without surgical resection of primary tumors and metastatic lung lesions. Herein, we report the synchronous metastasis of renal cell carcinoma to the bladder and lung.
\end{abstract}

Keywords: Carcinoma; Renal Cell; Urinary Bladder; Lung; Neoplasm Metastasis

\section{INTRODUCTION}

Renal cell carcinoma (RCC) can metastasize to almost every organ. The most common involved sites, in descending order of frequency, are the lung, lymph nodes, liver, contralateral kidney, and bone. Although the incidence of RCC continues to increase, bilateral tumors remain relatively infrequent, representing only $3 \%-5 \%$ of all cases [1]. The lungs are the most frequent site for metastases, and nearly $20 \%$ of all patients with RCC have pulmonary metastases when first diagnosed. However, urinary bladder metastasis is extremely rare, which can be synchronous [2] or metachronous [3], and less than $2 \%$ have been reported [1]. Herein we report a synchronous metastasis of RCC to the bladder and lung.

\section{CASE REPORT}

A 77-year-old woman presented to our hospital with a 2-day history of gross hematuria. She had Eastern Cooperative Oncology Group performance status 1 and laboratory examination showed anemia (hemoglobin $9.2 \mathrm{~g} / \mathrm{dl}$ ), hypocalcemia (total calcium $8.2 \mathrm{mg} / \mathrm{dl}$ ), and microscopic hematuria (urinalysis red blood cell 100/high power field). An abdominal and chest computed tomography (CT) showed a $8 \times 10 \mathrm{~cm}$ sized ill-defined heterogenic enhanced mass in the lower pole of the left kidney, $1.2 \times$ $1.0 \mathrm{~cm}$ sized enhanced bulging mass in the mid pole of the right kidney, $1.5 \times 0.8 \mathrm{~cm}$ sized enhanced polypoid mass in the dome of bladder, and multiple variable sized small round nodules in both lungs that suggested hematogenous metastases (Figure 1). Cystoscopy revealed a $1.2 \mathrm{~cm}$-sized submucosal tumor at the dome of the bladder wall. She had no known hereditary RCC syndrome and no symptoms and signs associated with renal mass. Therefore, we suspected synchronous sporadic bilateral RCC with lung metastasis and bladder cancer. We explained the current status and prognosis to the patient. She and family members did not consent to RCC surgical therapy but agreed to transurethral resection of the bladder tumor because of gross hematuria.

Subsequently, transurethral resection of the bladder tumor and sonography guided renal biopsy were performed in the left renal mass. Surprisingly, both pathology reports were clear cell RCC with Fuhrman nuclear grade 2 (Figures 2(A) and (B)). Bladder mass were concluded to be metastasis from RCC. Both of the tissues revealed positive reactivity for CD10 (Figures 2(C) and (D)) and vimentin (Figures 2(E) and (F)). However they revealed negative reactivity for uroplakin (Figures 2(G) and $\mathbf{( H ) )}$ and thrombomodulin.

The bladder mass was concluded to be metastasis from RCC. Targeted therapy using pazopanib was adminis- 


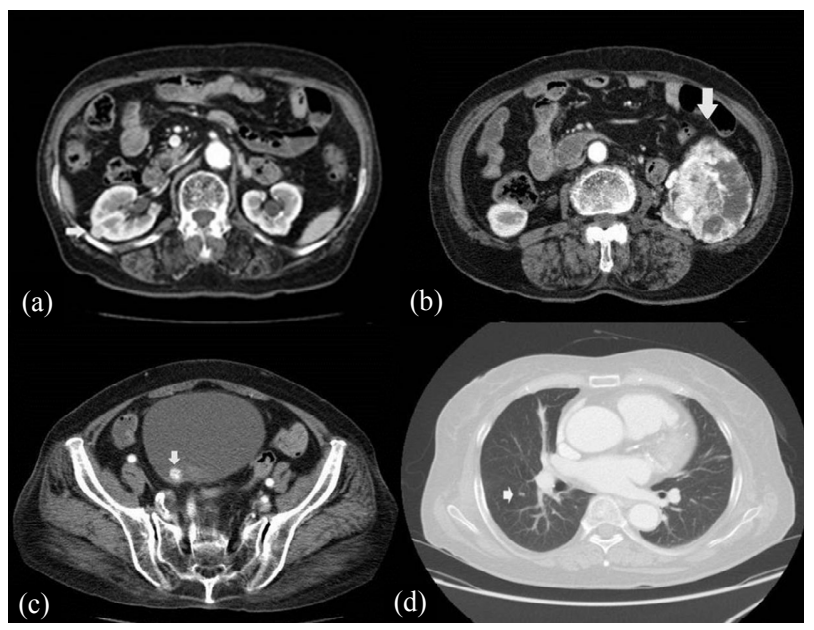

Figure 1. Pretreatment computed tomography scan findings. (a) A $1.2 \times 1.0 \mathrm{~cm}$ sized enhanced bulging mass in the mid-pole of the right kidney; (b) A $8 \times 10 \mathrm{~cm}$ sized ill-defined heterogenic enhanced mass in the lower pole of the left kidney; (c) A $1.5 \times$ $0.8 \mathrm{~cm}$ sized enhanced polypoid mass dome of bladder; (d) A lung metastatic nodule is denoted by the arrow.

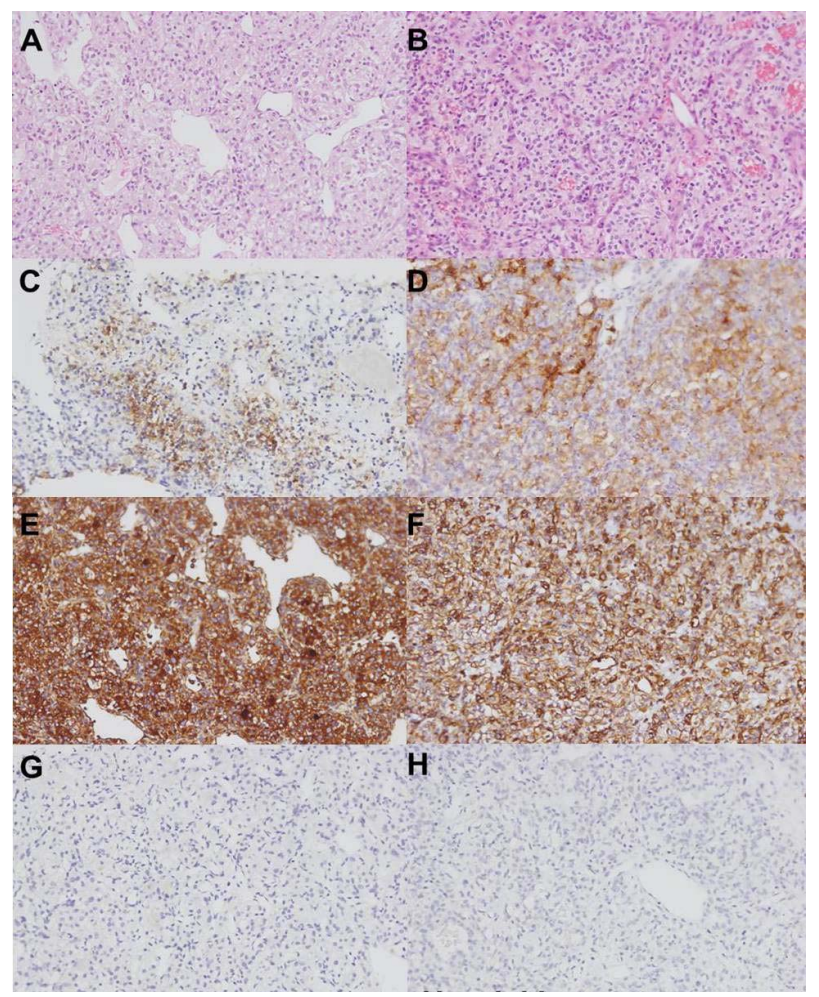

Figure 2. Microscopic examinations of kidney and bladder tumors. (A) Kidney tumor shows clear cell cytoplasm with large, irregular nuclei and prominent nucleoli (magnification $\times 100$ Hematoxylin and eosin stain); (B) Bladder tumor shows clear cell cytoplasm with large, irregular nuclei and prominent nucleoli (magnification $\times 100$ Hematoxylin and eosin stain). Both of the tissues revealed positive reactivity for CD10 and vimentin; ((C), (E): kidney; (D), (F): bladder) However they revealed negative reactivity for uroplakin $((\mathrm{G})$ : kidney; $(\mathrm{H})$ : bladder). tered. At the time of this report, 3 months after beginning of therapy, the patient remains alive.

\section{DISCUSSION}

Although the incidence of RCC continues to increase, bilateral tumors remain relatively infrequent, representing only $3 \%-5 \%$ of all cases [1]. A genetic or acquired predisposition towards bilateral and multifocal presentations has been established in patients with Von Hippel Lindau disease, familial renal carcinoma, and post-dialysis acquired cyst disease. Boorjian reported that $81 \%$ of all bilateral and metachronic renal tumors are accompanied by other common metastatic foci in lung, bone, or lymph nodes [4]. Thus, given the rarity of sporadic bilateral renal carcinoma, its management remains a challenge. In addition, urinary bladder metastasis is extremely rare, which can be synchronous [2] or metachronous [3] and less than $2 \%$ have been reported [1]. In terms of bladder metastasis from RCC, asynchronous metastasis is far more common than synchronous metastasis [5].

Although few case reports have described synchronous or metachronous solitary urinary bladder metastases in renal cell carcinoma, the combination of sporadic bilateral RCC and lung metastasis. Bilateral RCC with synchronous solitary urinary bladder metastasis has been described in only one VHL syndrome patient [5].

The mechanism of spread to the urinary bladder is controversial. Three different metastatic pathways have been suggested: hematogenous, lymphatic, and metastasis via the urinary stream. Hematogenous metastasis can occur through two different paths. One is dissemination through systemic circulation, and the other is retrograde metastasis through urethral vein or gonadal vein when the renal vein is obstructed by tumor thrombus [6]. An intriguing theory referred to as "drop metastases" posits direct extension and implantation [7]. Dissemination of neoplastic cells through the urinary tract is a valid way to explain anterograde spread of renal tumors to the ureter and bladder.

Management of bladder metastasis varied from transurethral resection and fulguration to partial cystectomy with or without ureterectomy. Less contemporary reports of bladder metastasis from RCC cite a dismal prognosis for these patients, with most dying less than 1 year following diagnosis of bladder metastasis [5]. More recent case reports have shown improved survival with resection of the metastatic lesion. Specifically, one case report described a patient with metachronous bladder metastasis who was alive and disease free at 6 years following transurethral resection [8]. Another case report described a patient with solitary synchronous bladder metastasis free of recurrence at 24 months [9]. Even though our patient was accompanied by lung metastases, resection of bladder metastasis is expected to extend survival. 
Swanson and Liles [10], recommended that cystoscopy should be performed in patients with RCC and hematuria. We agree with their careful evaluation of the urinary bladder. Recognition of this rare disease entity is important to prevent a delay in diagnosis and thereby improve the prognosis. Although in our case we could not resect the primary lesion and metastatic lesion, as has been reported, early detection of bladder metastasis can enable surgical resection of RCC that has metastasized to the bladder. We suggest that early detection and resection of bladder metastases is the best management strategy.

\section{REFERENCES}

[1] Marshall, F.F., Stewart, A.K. and Menck, H.R. (1997) The national cancer database: Report on kidney cancers. Cancer, 80, 2167-2174. doi:10.1002/(SICI)1097-0142(19971201)80:11<2167::AI D-CNCR18>3.0.CO;2-X

[2] Uygur, M.C., Özen, H.A., Sungur, A. and Remzi, D. (1994) A solitary and synchronous metastasis of renal cell carcinoma to the bladder. International Urology and $\mathrm{Ne}$ phrology, 26, 529-533. doi:10.1007/BF02767654

[3] Shiraishi K., Mohri J., Inoue R., et al. (2003) Metastatic renal cell carcinoma to the bladder 12 years after radical nephrectomy. International Journal of Urology, 10, 453455. doi:10.1046/j.1442-2042.2003.00652.x

[4] Boorjian, S.A., Crispen, P.L., Lohse, C.M., et al. (2008) The impact of temporal presentation on clinical and pathological outcomes for patients with sporadic bilateral renal masses. European Urology, 54, 855-863. doi:10.1016/j.eururo.2008.04.079

[5] Dogra, P., Kumar, A. and Singh, A. (2007) An unusual case of Von Hipple Lindau (VHL) syndrome with bilateral multicentric renal cell carcinoma with synchronous solitary urinary bladder metastasis. International Urology and Nephrology, 39, 11-14. doi:10.1007/s11255-006-6656-5

[6] Abeshouse, B.S. (1956) Metastasis to ureters and urinary bladder from renal carcinoma: Report of two cases. Journal of the International College, 25, 117-126.

[7] Négrier, S., Escudier, B., Gomez, F., et al. (2002) Prognostic factors of survival and rapid progression in 782 patients with metastatic renal carcinomas treated by cytokines: A report from the Groupe Francais d'Immunothérapie. Annals of Oncology, 13, 1460-1468. doi:10.1093/annonc/mdf257

[8] Raviv, S., Eggener, S.E., Williams, D.H., et al. (2002) Long-term survival after "drop metastases" of renal cell carcinoma to the bladder. Urology, 60, 697. doi:10.1016/S0090-4295(02)01846-0

[9] Dogra, P., Kumar, A. and Singh, A. (2007) An unusual case of Von Hipple Lindau (VHL) syndrome with bilateral multicentric renal cell carcinoma with synchronous solitary urinary bladder metastasis. International Urology and Nephrology, 39, 11-14. doi:10.1007/s11255-006-6656-5

[10] Swanson, D.A. and Liles, A. (1982) Bladder metastasis: A rare cause of hematuria in renal cell carcinoma. Journal of Surgical Oncology, 20, 80-82. doi:10.1002/jso.2930200204 